\title{
The Histopathological Difference between Oral Lichen Planus and Oral Lichenoid Mucositis in the Inflammation Involved by Innate Lymphoid Cells
}

\author{
Yang Gu${ }^{1 *}$, Ashley Kervin², Patricia Colp ${ }^{3}$, Tahani Osamah M Badeeb ${ }^{4}$ and Eran Maia Tsz Ying \\ Lam $^{5}$ \\ ${ }^{1}$ Assistant Professor, Oral Pathologist and Director of Oral Pathology Teaching Clinic, Department of Oral and Maxillofacial \\ Sciences, Faculty of Dentistry, Dalhousie University, Canada
}

${ }^{2}$ Medical Laboratory Technologist, Oral biopsy service laboratory, Department of Oral and Maxillofacial Sciences, Faculty of Dentistry, Dalhousie University, Canada

${ }^{3}$ Medical Laboratory Technologist and Laboratory Manager, Histology and Research Services Laboratory, Department of Pathology, Faculty of Medicine, Dalhousie University, Canada

${ }^{4}$ Teaching Assistant and General Dentist, Faculty of Dentistry, King Abdulaziz University, Canada

${ }^{5}$ Research assistant and general dentist, Canada

*Corresponding author: Yang Gu, Assistant Professor, Oral Pathologist and Director of Oral Pathology Teaching Clinic, Department of Oral and Maxillofacial Sciences, Faculty of Dentistry, Dalhousie University, Canada

\section{ARTICLE INFO \\ Received: 慧 April 21, 2020 \\ Published: May 14, 2020}

Citation: Yang Gu, Ashley K, Patricia C, Tahani Osamah M B, Eran Maia Tsz YL. The Histopathological Difference between Oral Lichen Planus and Oral Lichenoid Mucositis in the Inflammation Involved by Innate Lymphoid Cells. Biomed J Sci \& Tech Res 27(4)-2020. BJSTR. MS.ID.004533.

Keywords: Oral Lichenoid Reaction; Oral Lichen Planus; Oral Lichenoid Mucositis; Innate Lymphoid Cells and Immunohistochemistry Technique

\section{Abstract}

Objectives: To explore the role of innate lymphoid cells (ILCs) in the inflammation of oral lichenoid reactions (OLR) and to compare the difference between oral lichen planus (OLP) and oral lichenoid mucositis (OLM).

Methods: The patient-based case control study applied eight antibodies to check 74 biopsy specimens by the immunohistochemistry technique (IHC). Participants included 28 cases of oral lichen planus (OLP), 16 cases of contact stomatitis from dental restorative materials (OLM-dental) cases, 14 cases of mucosal reaction to systemic drug administration (OLM-drug), 15 cases of contact stomatitis from topical chemical exposure (OLM-contact) and one traumatic fibroma (TF) case. Eight antibodies wereanti-T-bet/Tbx21 (T-bet), anti-NKp46 (NKp46), anti-ST2 (ST2), anti-cytokine IL-7 receptor (IL-7R), anti-cytokine IL-22 (IL-22), anti-HAND2-Carboxyterminal End (HAND2), anti-cytokine IL-17 beta (IL-17B) and anti-interferon gamma (INFG).

Results: Innate lymphoid cells involve in the inflammation of OLR. The most significant involvement exists in contact stomatitis from topical chemical exposure (OLM-contact).

Abbreviations: ILCs: Lymphoid Cells; OLP: Oral Lichen Planus; OLM: Oral Lichenoid Mucositis; TF: Traumatic Fibroma; IHC: Immunohistochemistry; OLR: Oral Lichenoid Reaction

\section{Introduction}

Oral lichenoid reaction (OLR) refers to oral lichen planus (OLP) or oral lichenoid mucositis (OLM). Oral lichenoid mucositis could be one of three conditions, which include lichenoid contact stomatitis from dental restorative materials (OLM-dental), mucosal reaction to systemic drug administration (OLM-drug) and contact stomatitis from topical chemical exposure (OLM-contact). These four oral mucosal conditions share a similar clinical appearance of white reticular striations on the erythematous mucosal base with or without ulcerations. An incisional biopsy is required to confirm the definitive diagnosis [1] (Figure 1). However, it is still 
a challenge for oral pathologists to differentiate four types of oral lichenoid reactions histopathologically. Oral lichen planus (OLP) shows a band-like lymphohistiocytic infiltration immediately demolishing the basal cell layer of keratinized stratified squamous epithelium. Oral lichenoid mucositis (OLM) displays the similar histopathological features as OLP, but may have other features as well, which include patched lymphohistocytic infiltration and /or other inflammatory cells, such as plasma cells, eosinophils, and mast cells [2].
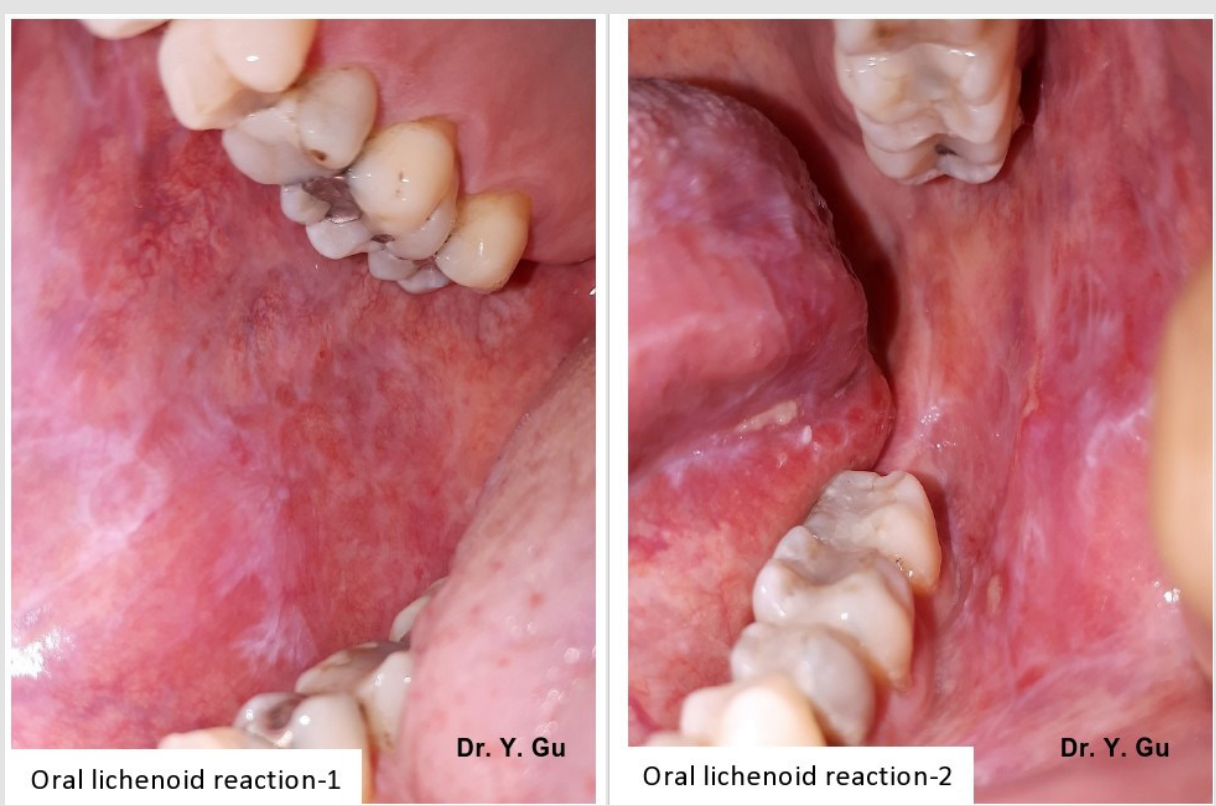

Figure 1: The clinical appearance of oral lichenoid reaction is white reticular striations in the erythematous mucosal base with or without ulcerations.

Nevertheless, there is no gold standard that can be followed (Figure 2). The pathogenesis of OLR is categorized in T-cell mediated hypersensitivity type IV. However, different pathways of four conditions are not clear. Innate lymphoid cells (ILCs) reside at mucosa surfaces and develop from lymphoid progenitors, but are absence of antigen specific B or T cell receptors [3]. ILCs work at epithelial barriers, interact with $\mathrm{T}$ cells and play as a regulator in inflammation and tissue homeostasis [4]. There are limited patient-based studies. The purpose of this case-control study based on human tissue specimens is to explore the exact pathway of each OLR. It will benefit for the histopathological diagnosis.

\section{Materials and Methods}

The case-control study used eight antibodies to check biopsy specimens of 28 OLP cases and 45 OLM cases by immunohistochemistry (IHC) technique. OLM cases included 16 OLM-dental, 14 OLM-drug and 15 OLM-contact specimens. In addition, a traumatic fibroma (TF) case was arranged into the study as a non-specific inflammation control. Participants aged between 28- and 72-year-old (average43) and with 82\% gender ratio (Female by Male) had lesions on buccal mucosa (64\%), gingival (35 $\%)$ or tongue (1\%). Eight antibodies are anti-T-bet/Tbx21 (T-bet), anti-NKp46 (NKp46), anti-ST2 (ST2), anti-cytokine IL-7 receptor (IL-7R), anti-cytokine IL-22 (IL-22), anti-HAND2-Carboxyterminal End (HAND2), anti-cytokine IL-17 beta (IL-17B) and anti-interferon gamma (INFG). We used a double-blind working protocol to manage the entire study. Specimens in four study groups were numbered alphabetically only by a histologist (AK) herself. The result was unveiled after the scoring and before the data analysis. For avoiding bias, we have positive controls, negative controls, a non-specific inflammation control, the crucial inclusion and exclusion standard in each step.

\section{Inclusion, Exclusion and Preparation}

A medical laboratory technologist (AK) searched OLP and/or OLM as key words in the database of Oral Biopsy Service (OBS) Laboratory of Faculty of Dentistry, Dalhousie University, and Halifax, Canada. Totally 296 cases were found between January $1^{\text {st }}, 2011$ and May 31, 2017. An oral pathologist (YG) read all H\&E slides. Only 87 OLP and OLM specimens plus one traumatic fibroma specimen were selected. Exclusion is ulcerative type of OLR, bullous type of OLR, OLR combined with fungus infection, inappropriate biopsy specimens and inadequate tissue blocks. The inclusive standard of study groups obeys to relatively specific histopathological features about OLP and OLM described in $4^{\text {th }}$ edition of Neville's oral and maxillofacial pathology. OLP showed band-like lymphohistiocytic infiltration immediately demolishing the basal cell layer of keratinized stratified squamous epithelium. OLM-dental showed the similar feature as OLP but has scattered plasma cells. OLM-drug showed the similar feature as OLP but has scattered eosinophils. OLM-contact showed the similar feature as OLP but has patched distribution (Figure 2). 


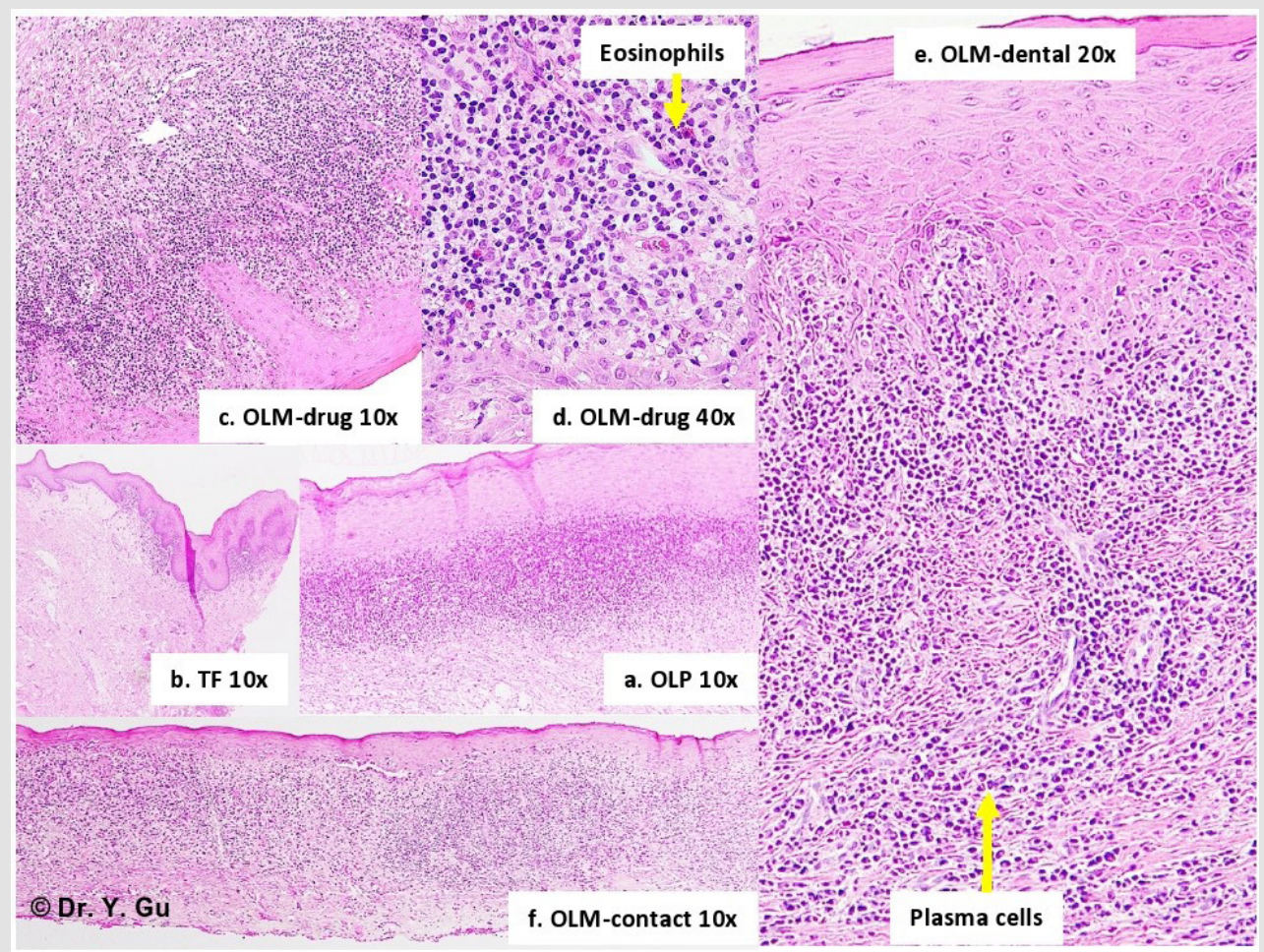

Figure 2: Histopathological features of oral lichenoid reactions

a. OLP (oral lichen planus): Band-like lymphohistiocytic infiltration immediately demolishing the basal cell layer of keratinized stratified squamous epithelium.

b. TF (traumatic fibroma): Dense fibrous connective tissue mass covered by keratinized stratified squamous epithelium with focally mild chronic inflammation.

c. OLM-drug (mucosal reaction to systemic drug administration): The similar feature as OLP, but has scattered eosinophils.

d. OLM-drug (mucosal reaction to systemic drug administration): The similar feature as OLP, but has scattered eosinophils.

e. OLM-dental (contact stomatitis from dental restorative materials): The similar feature as OLP, but has scattered plasma cells.

f. OLM-contact (contact stomatitis from topical chemical exposure): The similar feature as OLP, but has patched distribution.

The medical laboratory technologist (AK) sectioned specimens (4 um thickness, 3-5 mm length and 2-3 mm wideness) from paraffin tissue blocks, which were stored in the pathology archive of the OBS. She mounted 5-8 specimens on one glass slide. Therefore, 88 specimens were arranged in 14 slides. We named it "Minor-Array Plate". Totally 252 slides (14 x 18) were prepared for the IHC procedure. The process was conducted in the OBS laboratory. A chief medical laboratory technologist (PC) conducted the IHC manual staining procedure (Capillary Gap Technology) in the Histology and Research Service Laboratory of Faculty of Medicine, Dalhousie University, Halifax, Canada. The oral pathologist (YG) read all IHC slides. Eventually28 OLPand 45 OLM specimens plus one traumatic fibroma specimen were selected. Exclusion is uneven staining, unusual weak or absent staining, background or art factual staining and inadequate amount of a specimen.

\section{Antibodies and Optimal Titrated Dilution}

Eight primary antibodies and the antibody dilution buffer were purchased from Abcam (Toronto, ON, Canada M5W 0E9). Second antibody (mouse $\operatorname{IgG}$ ) and reagents were bought from Inter Medico (Markham, ON, Canada L3R 6E9). All antibodies are able of reacting to human tissue. We toned the optimal antibiotic dilutionin positive control specimens that were recommended by Abcam and published reference. Negative controls were obtained by replacing the primary antibody with mouse IgG (Table 1).

Table 1: Eight antibodies and optimal titrated dilutions.

\begin{tabular}{|c|c|c|c|c|c|c|c|c|}
\hline Antibody & T-bet & NKp46 & ST2 & IL7R & IL22 & HAND2 & IL17B & INFG \\
\hline $\begin{array}{l}\text { Abcam } \\
\text { number }\end{array}$ & Ab150440 & Ab214468 & Ab25877 & Ab118527 & Ab18499 & Ab60037 & Ab198891 & Ab9657 \\
\hline $\begin{array}{c}\text { Primary } \\
\text { antibody type }\end{array}$ & $\begin{array}{c}\text { Rabbit } \\
\text { Monoclonal }\end{array}$ & $\begin{array}{c}\text { Rabbit } \\
\text { Polyclonal }\end{array}$ & $\begin{array}{c}\text { Rabbit } \\
\text { Polyclonal }\end{array}$ & $\begin{array}{c}\text { Rabbit } \\
\text { Polyclonal }\end{array}$ & $\begin{array}{c}\text { Rabbit } \\
\text { Polyclonal }\end{array}$ & $\begin{array}{c}\text { Rabbit } \\
\text { Polyclonal }\end{array}$ & $\begin{array}{c}\text { Rabbit } \\
\text { Polyclonal }\end{array}$ & $\begin{array}{c}\text { Rabbit } \\
\text { Polyclonal }\end{array}$ \\
\hline
\end{tabular}




\begin{tabular}{|c|c|c|c|c|c|c|c|c|}
\hline $\begin{array}{l}\text { Positive } \\
\text { Control }\end{array}$ & Lymph Node & $\begin{array}{c}\text { Lung } \\
\text { Carcinoma }\end{array}$ & Placenta & Lymph Node & Tonsil & Mouse Heart & Rat Spleen & $\begin{array}{l}\text { Verrucous } \\
\text { Carcinoma }\end{array}$ \\
\hline $\begin{array}{l}\text { Cellular } \\
\text { Localization of } \\
\text { IHC staining }\end{array}$ & Nucleus & $\begin{array}{c}\text { Cell } \\
\text { Membrane }\end{array}$ & $\begin{array}{l}\text { Secreted\& Cell } \\
\text { Membrane }\end{array}$ & $\begin{array}{l}\text { Secreted } \\
\text { \& Cell } \\
\text { Membrane }\end{array}$ & Secreted & Nucleus & $\begin{array}{l}\text { Secreted \& cell } \\
\text { Membrane }\end{array}$ & Secreted \\
\hline $\begin{array}{l}\text { Optimal } \\
\text { titrated } \\
\text { Dilution }\end{array}$ & $1: 125$ & $1: 400$ & $1: 1600$ & $1: 100$ & $1: 250$ & $1: 50$ & $1: 200$ & $1: 500$ \\
\hline
\end{tabular}

Note: All primary antibodies react to human tissue. Positive controls are recommended by Abcam and published reference. Type of retrieval solution is Decloaker Citrate buffer solution (PH6.0). Second antibody is mouse probe. Detection system is MACH4 polymer and HRP polymer with DAB chromogen. Antibody dilution buffer was purchased in Abcam. T-bet: anti-T-bet/Tbx21; NKp46: antiNKp46; ST2: anti-ST2; IL-7R: anti-cytokine IL-7 receptor; IL-22: anti-cytokine IL-22; HAND2: anti-HAND2-Carboxyterminal End; IL-17B: anti-cytokine IL-17 beta; INFG: anti-interferon gamma.

\section{Immunohistochemistry Procedure}

Antigen retrieval was processed in the Biocare Autoclaveby PH 6.0 citrate buffer solution (DeCloaker) immediately after deparaffinization. The enzymatic digestion was conducted by Proteinase K. The procedure of protein block and endogenous enzyme block was achieved by Peroxidizer, Biocare's background Sniper, automation buffers (Triton-X-100 and Tween 20). The primary and secondary antibody were washed by automation buffer (1\% BAS and $0.025 \%$ Triton X-100) after enough incubation time respectively. The detection system was MACH 4 probe plus HRP-Polymer with DAB (diaminobenzidine) chromogen. The counterstain was Hematoxylin.

\section{Quantification of the Data and Scoring}

IHC slides were analyzed under an optical microscope (Olympus BX51 microscope; Olympus Optical Co., Tokyo, Japan) connected to a digital color camera/Q-Color 5 (Olympus). Images were obtained with10x, 20x and 40x objectives UPLanFI (resolution: $2.75 \mathrm{~mm}$ ), at a size of $2560 \times 1920$ pixels (resolution: $1 \mathrm{~mm}=3000$ pixels), under standard conditions. Pictures were taken from the whole slide to perform the scoring procedure. The proportion of immune-positive cells is used to account scores if the staining located in the nucleus, on the cellular membrane or within the endoplasmic reticulum. The combinative semi quantitative scoring is applied for the secreted staining. The scoring outcome will be the combination of two types of accounting methods if antibodies have two staining locations. The scoring principle is score $1:<10 \%$ and/or mild stain; score 2: $10-50 \%$ and/or mild-moderate stain; score 3: $50-90 \%$ and/or moderate-strong stain; score 4:>90\% and/or strong stain.

The scoring procedure was conducted by two researchers (TB and EL) and the oral pathologist (YG) respectively for 18 times. The scoring protocol is to score the staining outcome to epithelial region, sub epithelial region and sub mucosal region separately. Each case was marked by three scores. We called it "Sandwich Scoring" (Figure 3). The consistency of the immuno positive staining is different between study groups as well. Oral lichenoid mucositis (OLM) demonstrated a patched staining pattern. Oral lichen planus (OLP) addressed a band-like staining pattern. Traumatic fibroma showed a nest-like staining pattern. Those were recorded in the scoring data as well (Table 2).

Table 2: Mean values of IHC outcome in oral lichenoid reactions.

\begin{tabular}{|c|c|c|c|c|c|}
\hline Antibody & TF & OLP & OLM-dental & OLM-drug & OLM-contact \\
\hline T-bet & $4 / 1 / 0^{*}$ & $3 / 4 / 1$ & $3 / 4 / 1$ & $3 / 4 / 2$ & $4 / 4 / 1$ \\
\hline INFG & $1 / 1 / 0$ & $1 / 3 / 1$ & $1 / 3 / 1$ & $1 / 1 / 1$ & $1 / 2 / 1$ \\
\hline NKp46 & $3 / 1 / 0$ & $3 / 4 / 2$ & $3 / 4 / 2$ & $2 / 4 / 2$ & $3 / 4 / 2$ \\
\hline ST2 & $4 / 2 / 0$ & $3 / 4 / 1$ & $3 / 4 / 1$ & $3 / 4 / 2$ & $4 / 4 / 1$ \\
\hline IL-7R & $3 / 2 / 0$ & $3 / 4 / 1$ & $2 / 4 / 1$ & $2 / 4 / 2$ & $3 / 4 / 1$ \\
\hline IL-22 & $1 / 2 / 0$ & $2 / 4 / 1$ & $1 / 4 / 1$ & $2 / 4 / 2$ & $2 / 4 / 1$ \\
\hline HAND2 & $4 / 4 / 4$ & $4 / 4 / 4$ & $4 / 4 / 4$ & $4 / 4 / 4$ & $4 / 4 / 4$ \\
\hline IL-17B & $4 / 2 / 0$ & $3 / 4 / 1$ & $3 / 4 / 1$ & $3 / 4 / 2$ & $4 / 4 / 1$ \\
\hline $\begin{array}{c}\text { Immunopositive } \\
\text { distribution }\end{array}$ & Nest-like pattern & Band-like pattern & Patched pattern & Patched pattern & Patched pattern \\
\hline
\end{tabular}

*Scoring location: epithelial region/subepithelial region/submucosal region

Note: TF: traumatic fibroma; OLP: oral lichen planus; OLM-dental: lichenoid contact stomatitis from dental restorative materials; OLM-drug: allergic mucosal reaction to systemic drug administration; OLM-contact: contact stomatitis from topical chemical exposure (OLM-contact). T-bet: anti-T-bet/Tbx21; NKp46: anti-NKp46; ST2: anti-ST2; IL-7R: anti-cytokine IL-7 receptor; IL-22: anticytokine IL-22; HAND2: anti-HAND2-Carboxyterminal End; IL-17B: anti-cytokine IL-17 beta; INFG: anti-interferon gamma. 


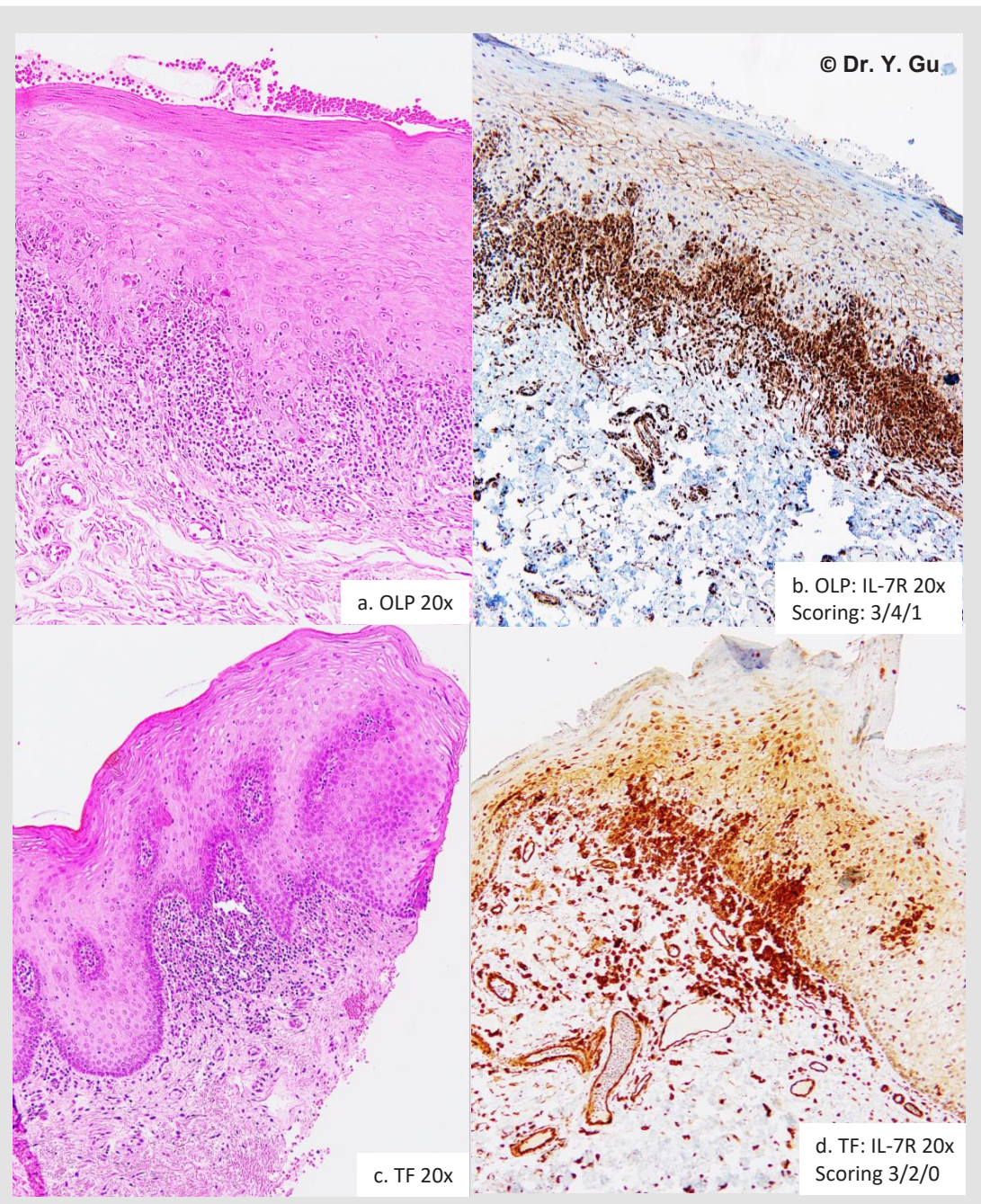

Figure 3: "Sandwich Scoring"

I. Scoring principle: Score 1: $<10 \%$ and/or mild stain; Score 2: 10-50\% and/or mild-moderate stain; Score 3: 50-90\% and/ or moderate-strong stain; Score 4: $>90 \%$ and/or strong stain.

II. Scoring protocol: Epithelial region; Sub epithelial region; Sub mucosal region.

a. OLP (oral lichen planus)

b. OLP IL-7R IHC scoring: Epithelial region 3; Sub epithelial region 4; Sub mucosal region 1

c. TF (traumatic fibroma)

d. TF IL-7R IHC scoring: Epithelial region 3; Sub epithelial region 2; Sub mucosal region 0.

\section{Analysis of the Data}

The study size are 28 cases in OLP group, 16 cases in OLMdental group, 14 cases in OLM-drug group and 15 cases in OLMcontact group. The outcome of immune positive scores in each group was obtained by the mean value of all cases. It is surprised that the standard deviation for the mean value in each group is one. It means specimens in each study group showed the same specific immune positive pattern. Therefore, we did the comparison and contrast based on their patterns, rather than on their individual data. We call it "Pattern Comparison". The comparing baseline is represented by the non-specific inflammation control. There is a significant difference if the mean score in a study group is higher or lower than the score in the same region in the non-specific inflammation control (Table 2).

\section{Result}

Epithelium always plays a role of mediators and regulators in immune response [5]. Available molecular signals of epithelial stability and immune response are HAND2, IL-17B, T-bet, INFG, ST2 and IL-17R. We received all immune positive outcomes in four study groups (Table 2 \& Figure 4). Traumatic fibroma (TF) was used as a baseline control for the comparison. There was no difference between four study groups and traumatic fibroma case in the immune positive staining of HAND2, which represents the epithelial stability. The OLM-contact group didn't show any abnormality in the epithelial region of all signals. The OLP group presented with three weaker staining of T-bet, ST2 and IL-17B. The OLM-dental and OLM-drug groups displayed four weaker staining of T-bet, ST2, IL-17R and IL-17B. The weakness of epithelial immune response 
is prominent in OLM-drug cases and OLM-dental cases, following with OLP, while OLM-contact demonstrated a normal epithelial immune response.

Common molecular signals of innate lymphoid cells (ILCs) are T-bet, NKp46, INFG, ST2, IL-7R and IL-22 [3]. ILCs group 1 (ILC1) is labelled by T-bet, NKp46 and INFG. ILCs group 2 (ILC2) is marked by ST2. ILC3 group 3 (ILC3) is tagged by IL-7R and IL22 [4]. We received all immune positive outcomes in four study groups. (Table 2 \& Figure 5). Traumatic fibroma (TF) was used as a baseline control for the comparison. There is a significant increase of all signals in the sub epithelial region of four study groups. The stronger expression of IL-22 in the epithelial region of OLP, OLMdrug and OLM-contact groups were noticed. The weaker expression of NKp46 in the epithelial region of OLM-drug group was prominent. Combined with the result of the epithelial immune response in four study group. The intensity of ILCs involved inflammatory process may be arranged in a descending order of OLM-contact group, OLP group, OLM-dental group and OLM-drug group.

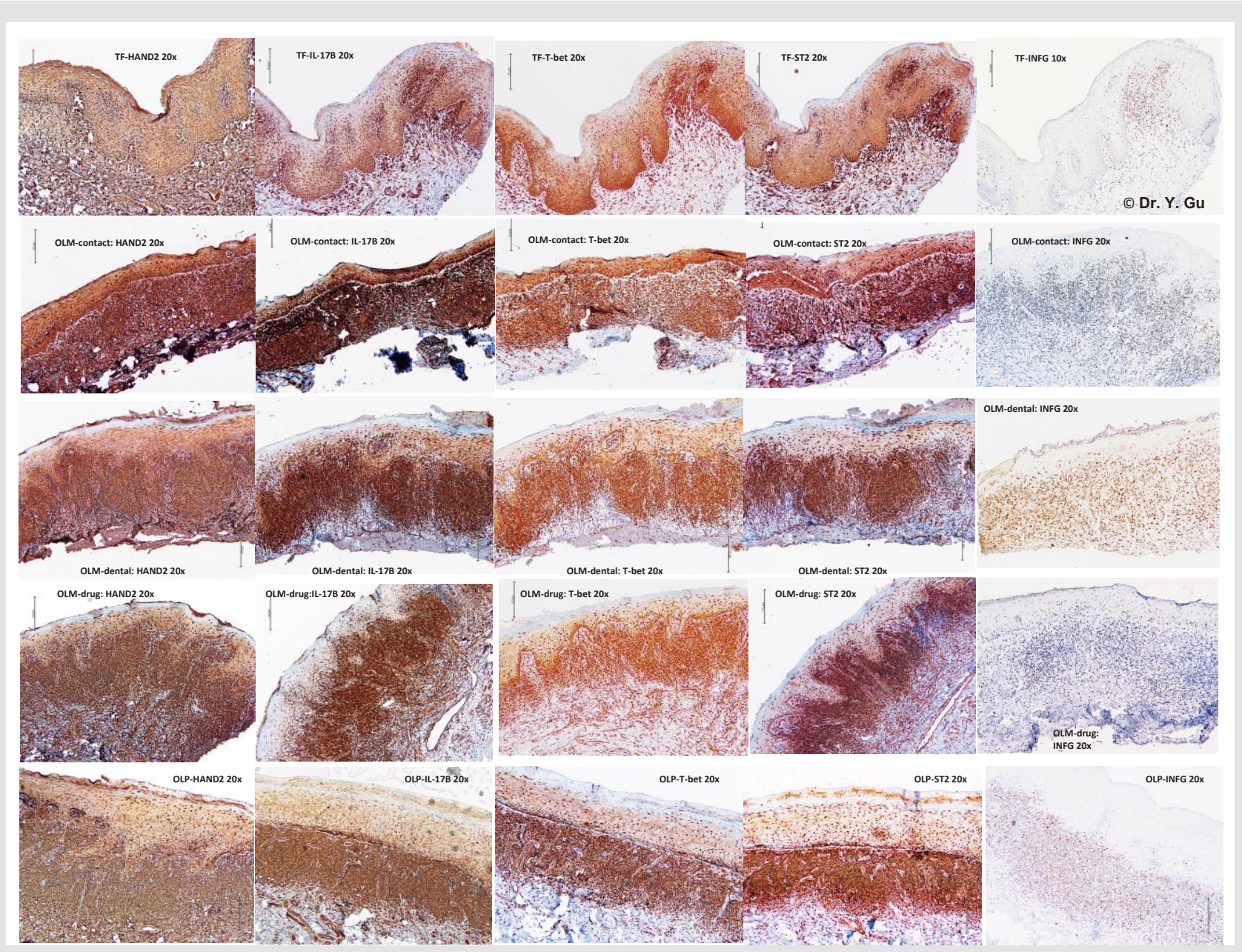

Figure 4: Immunohistochemistry (IHC) outcome of oral lichenoid reactions regarding to antibodies of HAND2, IL-17B, T-bet, ST2 and IFNG. Four study groups: oral lichen planus (OLP); mucosal reaction to systemic drug administration (OLM-drug); contact stomatitis from dental restorative materials (OLM-dental); contact stomatitis from topical chemical exposure (OLMcontact). Non-specific inflammation control: traumatic fibroma (TF). Five IHC antibodies: anti-HAND2-Carboxyterminal End (HAND2), anti-cytokine IL-17 beta (IL-17B), anti-T-bet/Tbx21 (T-bet), anti-ST2 (ST2), and anti-interferon gamma (INFG).

\section{Discussion}

Heart-and neural crest derivatives-expressed protein 2 (HAND2) is encoded by HAND2 gene and is a transcription factor plays an important role in heart, limb and branchial arch development through Sonic Hedgehog (SHH) pathway [www. genecards.org]. A recent research found it reduced expression in breast cancer [6]. There is no significant difference between traumatic fibroma and four study groups. This result provided evidence that all study specimens didn't have a developmental abnormality and a malignant potential. T-bet is a transcription factor encoded by TBX21 gene involving in developmental process, especially in the Th1 lineage and controlling the INFG expression [www.genecards.org]. T-bet is considered as a molecular signal of ILC1. T-bet was found expression on the epithelium of reproductive tract [7]. There is a significant increase of T-bet in the sub epithelial region of four research groups, while the weaker expression of T-bet on the oral mucosal epithelium of OLP, OLM-dental and OLMdrug groups was detected. It may indicate the status of epithelial immune response. 
NKp46 is encoded by NCR1 gene and related to pathways of major histocompatibility complex (MHC) I presentation and ILCs differentiation [www.genecards.org]. NKp46 mainly expresses on NK cells and NK-T cells [8]. There is a significant increase of NKp46 in the sub epithelial region of four study groups, while the OLM-drug group displayed a weaker expression of NKp46 in the epithelial region. It may consider that OLM-drug cases less likely involve in the cytotoxicity process by NK cells and NK-T cells. Interferon gamma (INFG) is encoded by INFG gene and classified by ontology as cytokine activity [www.genecards.org]. INFG is produced by inflammatory cells and mucosal epithelium. There was no difference between traumatic fibroma and four study groups in the epithelial expression of IFNG. In addition, IFNG promotes the differentiation first, and then Th1 produces INFG consequently [9]. The weakness of INFG expression in the sub epithelial region of OLM-drug group implies Th1 less likely involves in the OLM-drug inflammation.
Serum stimulation-2 (ST2) is encoded by IL-1 receptor like 1 (IL1RL1) gene and involved in ILCs differentiation [www. genecards.org]. ST2 is IL-33 receptor expressed on inflammatory cells and epithelial cells and possibly involves in Th2 function [10]. There is a significant increase of ST2 in the sub epithelial region of four study groups, while the weaker expression of ST2 in the epithelial region of OLP, OLM-dental and OLM-drug groups was prominent. It may specify the lower status of epithelial immune response. IL-7R is encoded by IL-7R gene and plays a critical role $\mathrm{V}$ (D) J recombination during lymphocyte development [www. genecards.org]. IL-7R expresses on lymphocytic precursors, ILC3 and antigen presenting cells [11]. There is a significant increase of IL-7R in the sub epithelial region of four study groups, while the lower expression of IL-7R in the epithelial region of OLM-dental and OLM-drug group was prominent. It may indicate ILC3 less likely affecting in OLM-dental and OLM-drug cases.

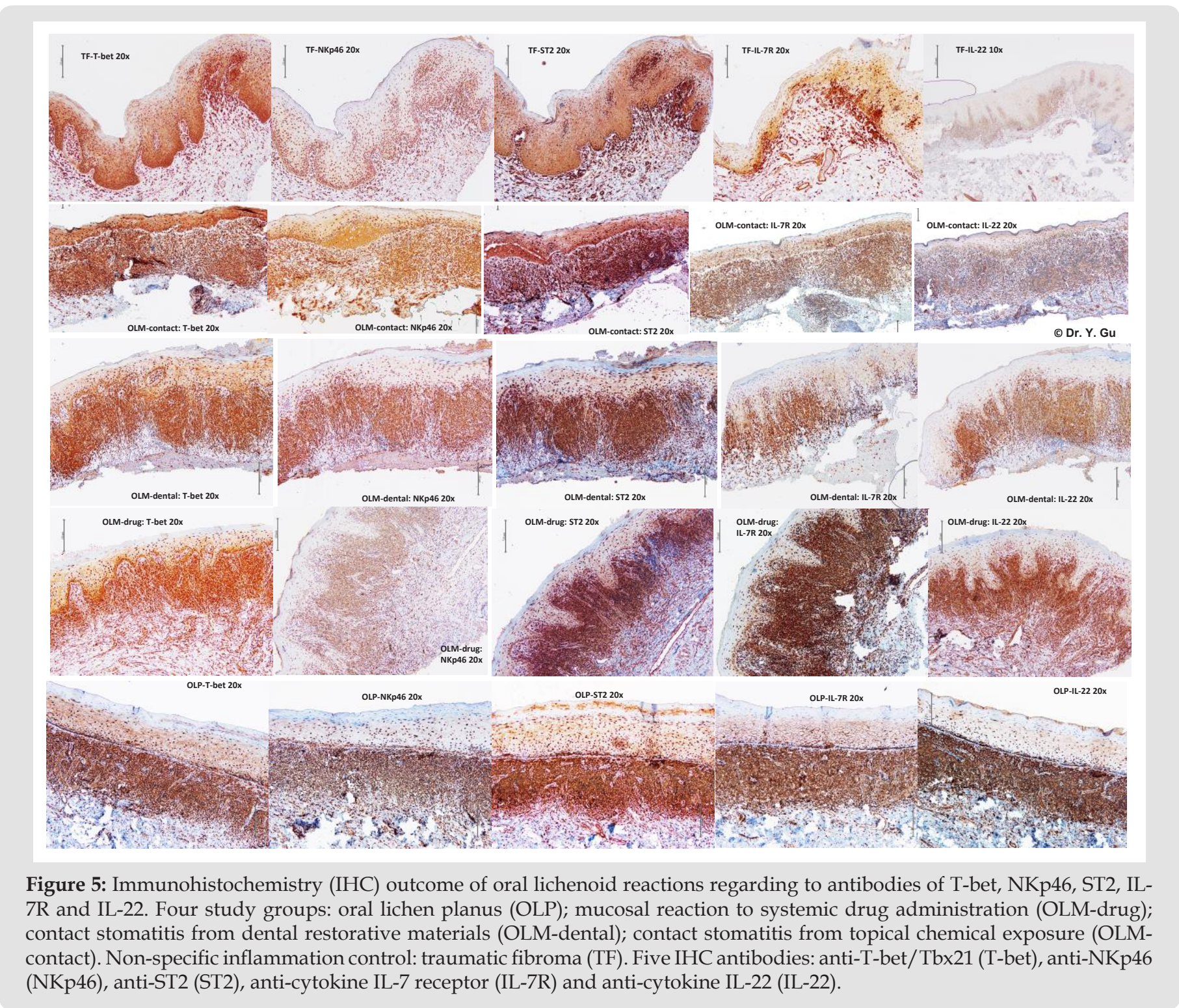

Copyright@ Yang Gu | Biomed J Sci \& Tech Res | BJSTR. MS.ID.004533. 
IL-22 is encoded by IL-22 gene and classified by ontology as cytokine activity [www.genecards.org]. Th17 cells and ILC3mainly produce IL-22, while the IL-22 receptor expresses on epithelial and stromal cells. The receptor-ligand interaction leads to activation of the transcription factor STAT3 in the target cell [12]. There is a significant increase of IL-22 in the sub epithelial region of four study groups, while the stronger expression of IL22 in the epithelial region of OLP, OLM-drug and OLM-contact groups was identical. It may implicit Th17 and ILC3 involved in their inflammatory process. IL-17B is encoded by IL-17B gene and classified by ontology as cytokine activity [www.genecards.org]. IL-17B stimulates monocytes to produce TNF-alfa and IL-1 beta, while epithelial cells and inflammatory cells produce IL-17B [13]. IL-17B has 29\% similarity with IL-17A, which produced by Th17. There is a significant increase of IL-17B in the sub epithelial region of four study groups, while the weaker expression of IL-17B in the epithelial region of OLP, OLM-dental and OLM-drug groups was notable. It may imply the abnormality of their immune response.

\section{Conclusion}

1. Innate lymphoid cells (ILCs) involve in the inflammatory process of oral lichenoid reaction (OLR), but different types of OLR have different levels of involvement.

2. Contact stomatitis from topical chemical exposure (OLMcontact) doesn't show any abnormality of epithelial immune response in molecular markers of T-bet, ST2, IL-7R and IL17B. It indicates ILCs is fully and significantly involved in its inflammation.

3. The inflammation of lichenoid contact stomatitis from dental restorative materials (OLM-dental), mucosal reaction to systemic drug administration (OLM-drug) and oral lichen planus (OLP) are partially involved in by ILCs.

\section{Conflicts of Interest}

All authors declare they have no conflict of interest.

\section{Ethical Approval}

All procedures performed in this study were in accordance with the principles of the Tri-Council Policy Statement Ethical Conduct for Research Involving Humans. This study was approved by Health Sciences Research Ethics Board of Dalhousie University with REB number 2016-4020.

\section{Funding}

This study was funded by Faculty of Dentistry Research Grant of Dalhousie University (No. 36650 and No. 34899).

\section{References}

1. Yi Shing Lisa Cheng, Alan Gould, Zoya Kurago, John Fantasia, Susan Muller (2016) Diagnosis of oral lichen planus: a position paper of the American Academy of Oral and Maxillofacial Pathology. Journal of oral surgery, oral medicine, oral pathology and oral radiology 122(3): 332354.

2. Brad W Neville, Douglas D Damm, Carl M Allen, Angela C Chi (2016) Oral and Maxillofacial pathology ( $4^{\text {th }}$ Edn.). Elsevier Inc. St. Louis, Chapter 9: 317-326 and Chapter 16: 729-734

3. Jennifer K Bando, Marco Colonna (2016) Innate lymphoid cell function in the context of adaptive immunity. Nature Immunology 177(7): 283289.

4. Christoph SN Klose, David Artis (2016) Innate lymphoid cells as regulators of immunity, inflammation and tissue homeostasis. Nature Immunology 17(7): 265-274.

5. Robert P Schleimer, Atsushi Kato, Robert Kern, Douglas Kuperman, Pedro C Avila (2007) Epithelium: At the interface of innate and adaptive immune responses. Journal of Allergy Clinical Immunol, 120(6): 12791284

6. Quiti dos Anjos Lopes, Ernesto De Paula Guedes neto, Luiza Azevedo Gross, José Luiz Pedrini, Ricardo Francalacci Savaris (2019) Heart and Neural Crest Derivatives Expressed Transcript 2 (HAND2) is Reduced in Women with Breast Cancer, A Case Control Study. Clinical Oncology \& Research 2(5): 1-6.

7. K Kawana, Y Kawana, RK Ashby, RN Patel, LH Glimcher, et al. (2004) T-bet a key transcriptional factor for Th1 development, is expressed and regulated via Stat family in the epithelial cells of the reproductive tract. Fertility and Sterility 82(2): S59.

8. Jianhua Yu, Takeki Mitsui, Min Wei, Hsiaoyin Mao, Jonathan P Butchar, et al. (2011) NKp46 identifies an NKT cell subset susceptible to leukemic transformation in mouse and human. Journal of Clinical Investigation 121(4): 1456-1470.

9. Vinay Kumar, Abul K Abbas, Nelson Fausto, Jon C Aster (2010) Robbins and Cotran Pathologic Basis of Disease ( $8^{\text {th }}$ Edn .). Saunders Elsevier Inc. Philadelphia, USA, Chapter 6: 205-208.

10. Brad Griesenauerand, Sophie Paczesny (2017) The ST2/IL-33 axis in immune cells during inflammatory diseases. Frontiers in immunology 8(475): 1-17

11. Renata I Mazzucchelli, Agostino Riva, Scott K Durum (2012) The human IL-7 receptor gene: deletions, polymorphisms and mutations. Seminars in Immunology 24: 225-230.

12. Lauren A Zenewicz (2018) IL-22: There is a gap in our knowledge. ImmunoHorizons 2(6): 198-207.

13. Qingli Bie, Chengqiang Jin, Bin Zhang, Haixin Dong (2017) IL-17B: A new area of study in the IL-17 family. Molecular Immunology 90: 50-56. 
ISSN: 2574-1241

DOI: 10.26717/BJSTR.2020.27.004533

Yang Gu. Biomed J Sci \& Tech Res

(C) (i) This work is licensed under Creative

Submission Link: https://biomedres.us/submit-manuscript.php

$\begin{array}{ll}\text { BIOMEDICAL } & \text { Assets of Publishing with us } \\ \text { RESEARCHES } & \text { - Global archiving of articles } \\ & \text { - Immediate, unrestricted online access } \\ & \text { - Rigorous Peer Review Process } \\ \end{array}$

